\title{
WNEREFACETEENOLOGGCA
}

\section{UM ESTUDO SOBRE GESTÃO POR PROCESSOS NA PRODUÇÃO INDUSTRIAL}

\section{A STUDY ON PROCESS MANAGEMENT IN INDUSTRIAL PRODUCTION}

\author{
Antonio José de Moura Junior - juninhomoura2010@ hotmail.com \\ Ramilio Ramalho Reis Filho - Ramilio.ramalho@hotmail.com \\ Faculdade de Tecnologia de Taquaritinga (FATEC) -SP -Brasil
}

DOI: 10.31510/infa.v16i2.674

\begin{abstract}
RESUMO
Gestão por processos é um conceito importante e de grande abrangência sendo bem abordado em meio acadêmico e em organizações, com a necessidade de se manter os processos com total qualidade para a obtenção de um melhor produto final diminuindo custos desnecessários e tendo a aprovação no mercado, as empresas estão adotando a utilização de novos modelos de gestão de processos. Partindo destes princípios o objetivo do presente artigo é abordar e analisar de maneira holística a importância, tipos e modelos da gestão por processos para o enaltecimento de empresas, podendo proporcionar ganhos na qualidade final do produto, eficácia e produtividade nas empresas, aferindo de modo a minimizar o tempo de reposta do processo evitando desperdício de tempo e garantindo que a informação chegue sem distorção, tendo isso como um diferencial em meio aos seus concorrentes diretos e indiretos proporcionando um emolumento à organização.
\end{abstract}

Palavras-chave: Gestão por processos. Total qualidade. Produto final.

\begin{abstract}
Process management is an important and wide-ranging concept that is well addressed in academia and organizations, with the need to maintain full quality processes to achieve a better end product while reducing unnecessary costs and having market approval, Companies are adopting new process management models. Based on these principles, the objective of this paper is to approach and analyze in a holistic way the importance, types and models of process management for the enhancement of companies, and can provide gains in the final product quality, efficiency and productivity in companies, measuring in order to minimize process response time avoiding wasted time and ensuring that information arrives without distortion, having it as a differentiator among its direct and indirect competitors providing a fee to the organization.
\end{abstract}

Keywords: Process management. Total quality. Final product. 


\section{WTERPFACETEENOLOLGCA}

\section{INTRODUÇÃO}

“A gestão por processos refere-se a um conjunto de funções de planejamento, direção e avaliação das atividades sequenciais, com a finalidade de minimizar os conflitos interpessoais e atender as necessidades e expectativas dos clientes externos e internos das empresas." (OLIVEIRA, 2011).

Partindo destas definições, para que uma Indústria obtenha sucesso em suas operações é necessário que se tenha controle e alinhamento na gestão dos processos da organização garantindo a eficácia em suas operações.

De modo natural a gestão por processos acompanhou os avanços e necessidades das organizações, e procura envolver todos os setores da empresa, garantindo decisões organizadas e sem distorção na comunicação.

"O modelo de gestão não pode se basear em comando e controle: precisa de negociação e colaboração.” (HAMMER E STANTON, 1999).

“A essência da gestão por processo é a coordenação das atividades realizadas na empresa." (GONÇALVES, 1997).

\subsection{Objetivo}

O objetivo do presente artigo é abordar e analisar a importância da gestão por processos para o enaltecimento de empresas, proporcionando ganho na qualidade final do produto, eficácia e produtividade nas empresas, aferindo de modo a minimizar o tempo de reposta do processo evitando desperdício de tempo e garantindo que a informação chegue sem distorção, proporcionando um emolumento à organização.

\subsection{Justificativa}

Por ser um tema que se torna cada vez mais relevante levando em consideração a necessidade das Industrias se diferenciarem no mercado.

Segundo Yin (1994) existem três condições básicas para a escolha da estratégia de pesquisa, independentemente da finalidade de esta ser exploratória, descritiva ou explanatória, mesmo que a fronteira entre as estratégias como experimentos, pesquisa de campo, análise de arquivo, histórico e estudos de casos, não seja clara e bem definida. As condições são: 


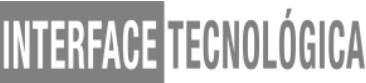

- O tipo de questão básica da pesquisa (como, o que, onde, quando e quais).

- A extensão do controle que o investigador tem sobre os eventos comportamentais (não controle).

- O grau de ênfase em eventos contemporâneos como oposto a eventos históricos (ênfase na situação atual). Na primeira etapa da pesquisa foi desenvolvido um questionário para coleta de dados, foi aplicada uma série de perguntas que devem ser respondidas com a presença do entrevistador na empresa em que foi feito o estudo de caso.

\section{REVISÃO DA LITERATURA}

“A gestão por processos, ou Business Management Process (BPM), é uma disciplina de gestão que exige das organizações uma mudança para o pensamento centrado nos processos" (DOEBELI et. al., 2011).

“A Gestão por Processos pode ser entendida como todos os esforços de uma organização em analisar e continuamente melhorar suas atividades fundamentais “(TRKMAN, 2010).

“A ideia central por trás BPM é que as organizações devem coordenar os esforços de seus processos e gerencia-los como um ativo" (HARMON e WOLF, 2012).

De acordo com Hammer e Champy (1994) processo é um grupo de atividades realizadas numa sequência lógica com o objetivo de produzir um bem, ou um serviço que tem valor para um grupo específico de clientes.

\subsection{Gestão por processos}

“A abordagem é baseada em uma arquitetura de processos que captura as interrelações entre os processos-chave de negócios, juntamente com a habilitação de processos de apoio e seu alinhamento com as estratégias, metas e políticas de uma organização" (ARMISTED e PRITCHARD, 1999).

De acordo com Paim, Cardoso e Caulliraux (2009) Muitas empresas consultores e pesquisadores destacam o modelo de estruturação organizacional, fundamentado na gestão funcional centrada na especialização e na delegação da coordenação para a hierarquia. 


\section{WNTEPHAGETEENOLLGGCA}

“A limitação da gestão completamente funcional e a necessidade de coordenação das atividades reforçam a busca por formas e mecanismos para gerenciar os processos, a rápida inovação dos processos pode resultar em capacitações organizacionais” (KANTER, 1995).

\subsection{Organização por processos}

"Identificar o processo como sendo a maneira típica de realizar o trabalho é importante para definir a forma básica de organização das pessoas e dos demais recursos da empresa." (DREYFUSS, 1996).

“As empresas estão procurando se organizar por processos para terem maior eficácia na obtenção do seu produto ou serviço, melhor adaptação, melhor integração de seus esforços e maior capacidade de aprendizado." (GONÇALVES, 1997).

\subsection{Tipos de Processo}

A seguir será explanado sobre as tipologias de processos e suas características dentro de uma indústria, de modo a integralizarem entre si levando a compreensão da importância em que esses processos tem de modo direto no desempenho industrial.

\subsubsection{Processos primários}

Processos primários são aqueles que tendem a gerar serviços ou produtos para algum público externo especifico. Por meio deles, as organizações atendem às necessidades de seus clientes.

\subsubsection{Processos de apoio}

Processos de apoio ou suporte são aqueles processos que não têm relação com público externo. O principal objetivo é fortalecer ou auxiliar os processos primários.

De forma natural, processo de apoio está ligado a áreas que afetam processos primários. 


\section{WTERPAREETEENOLOGGCA}

\subsubsection{Processos gerenciais}

Processo gerencial é aqueles que esta ligada à gestão estratégica da empresa. Por isso, estão relacionados aos outros dois tipos descrevidos anteriormente, é partir desse tipo de processo que as demais atividades podem ser continuamente monitoradas e melhoradas, processos gerenciais ajudam a trazer mais eficácia a empresa.

\subsection{Etapas na gestão por processos de Projeto}

Na sequência será apresentada as etapas primordiais na gestão por processos de um projeto, etapas essas indispensáveis em qualquer produto ou serviço fornecido.

- Projeto

- Mapeamento

- Simulação

- Execução

- Monitoramento

- Melhoria

\subsubsection{Projeto}

“Gerenciar projetos de forma eficiente em um contexto de constantes mudanças é um dos grandes desafios do executivo dos tempos modernos" (KERZNER, 2001).

Segundo Prado (2000) a boa prática de gerenciamento de projetos produz resultados expressivos para as organizações como:

- Redução no custo e prazo de desenvolvimento de novos produtos;

- Aumento no tempo de vida dos novos produtos;

- Aumento de vendas e receita;

- Aumento do número de clientes e de sua satisfação;

- Aumento da chance de sucesso nos projetos.

Consiste no planejamento de como a gestão deste processo será realizada na empresa, neste período é realizado um laudo listando para verificar as principais barreiras e qual a melhor forma em que essas barreiras podem ser ultrapassadas utilizando a gestão por 


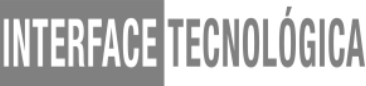

processos, este é o momento do projeto em que é definido o método, meta, notação, e ferramentas, que são necessárias durante todo ciclo.

\subsubsection{Mapeamento}

Slack et al. (2009) descreve que mapeamento de processo é a descrição de como os processos e as atividades relacionam-se, mostrando o fluxo de materiais, pessoas e informações, permitindo a compreensão de todo o macroprocesso, que geralmente é representado graficamente para facilitar o entendimento.

Os processos são levantados, detalhados no conjunto de atividades que os compõem e validados para garantir que o seu entendimento está correto.

Neste período, o processo da empresa é mapeado, de modo primordial é necessário que se conheça a organização como um todo. Para isso, é necessário que se desenhe a sua cadeia de valor, aonde são especificados os principais processos da empresa. Esses processos são normalmente classificados em três tipologias: gestão, apoio ou suporte e negócio.

Após a cadeia de valor ser definida, os processos são priorizados para que se inicie o levantamento do processo atual. Neste momento, são realizadas entrevistas registradas em documentos de anotações ou aplicadas outras técnicas de levantamento para mapear, com os principais responsáveis pelos processos. Após esse levantamento são desenhados os fluxos dos processos atuais.

\subsubsection{Simulação}

Os processos são simulados normalmente com base em medidas de desempenho.

Neste período, são planejados os resultados estimativos que o processo deve alcançar, posteriormente, se utiliza uma ferramenta de simulação, o processo é testado para verificar se o mesmo ele está sendo executado da maneira prevista ou se estão ocorrendo possíveis filas ou gargalos. Caso sejam detectados problemas, o processo pode ser alterado antes de ser disponibilizado para uso efetivo dentro da empresa. Quando o comportamento do processo estiver de acordo com o esperado, então ele poderá ser utilizado na prática.

\subsubsection{Execução}




\section{WNTEPHAGETEENOLLGGCA}

É primordial e necessário fazer o planejamento de como será feita essa implantação, levando em consideração necessidades e treinamentos, auxilio de software, implantando novas tecnologias e sistemas, mudando a estrutura organizacional, deslocando e remanejando equipes de colaboradores.

A gestão vai auxiliar a organização nas mudanças necessárias de modo a que o impacto resultante seja minimizado e que não interfira tanto na rotina dos colaboradores.

Essas mudanças devem ser vistas por parte dos colaboradores como algo positivo que venha agregar levando ao crescimento da empresa.

\subsubsection{Monitoramento}

Neste período os processos são acompanhados através de indicadores de desempenho definidos para verificar se houve algum desvio

Caso esse processo atual não alcance os resultados teóricos esperados, é necessário aderir a ações para controle dos desvios observados.

\subsubsection{Melhoria}

Neste período a situação real dos processos é estudada e avaliada, ideias de melhorias são direcionadas para que processos futuros sejam modelados

É no período de melhoria que ocorre a avaliação da situação real, para que seja proposta melhorias nos processos futuro de acordo informações levantadas no período de monitoramento.

Essas melhorias são correspondias à inclusão de ideias no processo, modificação no processo, retirada de etapas do processo.

\subsection{Visão sistêmica}

"O termo "sistema" refere-se um "conjunto unitário", como por exemplo, uma organização, que é composta de partes, e que trabalha dentro de um sistema maior que no caso de empresas modernas é a rede de alimentação ou de cadeia” (PIERCY et. al., 2009). 


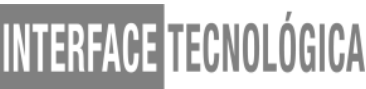

"O pensamento sistêmico tem desenvolvido uma dinâmica de sistemas e além, de modo a incluir as teorias da emergência e da complexidade, como a teoria de sistemas complexos adaptativos." (JACKSON, 2000).

Segundo Bider et. al. (2011), o pensamento sistêmico faz sentido por meio de três conceitos:

- A inter-relação: o conceito de como as coisas está ligadas e quais as consequências destas relações. O conceito de inter-relações abrange aspectos dinâmicos, da natureza não linear, do emaranhado, do contexto das estruturas de relacionamento, dos processos e dos padrões;

- O das perspectivas: o conceito de como enxergar os cenários. Olhar os cenários por diferentes aspectos e usando diferentes conjuntos de suposições dos stakeholders (Publico estratégico), valores e visões de mundo;

- O dos limites: o conceito de que não é possível pensar em tudo, colocar limites ao redor do pensamento não é opcional. Abordagem de questões práticas e éticas de onde e como está vinculado o foco de investigação.

Visão sistêmica pode ser definida como a capacidade em que o gestor tem e que o permite analisar a empresa como um todo, e através desta analise conseguir identificar e melhorar os processos, assim como os processos que o integram.

Também envolve diretamente entender a relação do ambiente interno com o ambiente externo, sendo assim o profissional que consiga absorver essas habilidades é visto como alguém que tem uma visão sistêmica.

\section{TÉCNICAS DE PESQUISA}

Neste artigo foi adotado como metodologia para pesquisa e desenvolvimento, foi realizado uma revisão com foco bibliográfico, se baseando em artigos de relevância acadêmica a fim de explorar e compreender de forma técnica a gestão por processos.

"A pesquisa de natureza aplicada produz conhecimentos aplicados na prática e dirigidos à solução de problemas.” (FREITAS; PRODANOV, 2013).

Yin (2001) distingue a pesquisa histórica como aquela com a capacidade de lidar com o passado, ou seja, quando não existem pessoas vivas para expor o que realmente aconteceu e, sendo assim, o pesquisador deve confiar em documentos, além de artefatos físicos e culturais. 


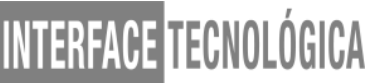

“As pesquisas exploratórias são compreendidas como investigações de pesquisa empírica cujo objetivo é a formulação de questões ou de um problema, com tripla finalidade: desenvolver hipóteses, aumentar a familiaridade do pesquisador com um ambiente, fato ou fenômeno para a realização de uma pesquisa futura mais precisa ou modificar e clarificar conceitos.” (LAKATOS E MARCONI 2001).

Após definir o tipo de pesquisa a ser feita foram estabelecidos critérios para que o artigo pudesse enriquecer em conteúdo bibliográfico.

Tabela 1 - Critérios para seleção dos artigos

\begin{tabular}{c|c}
\hline Artigos em potencial & $\begin{array}{c}\text { Todos os artigos que contribuem para o tema, } \\
\text { gestão por processos. }\end{array}$ \\
\hline Publicações entre 2010 e 2019 & $\begin{array}{c}\text { Para que o presente artigo seja desenvolvido foi } \\
\text { adotado como bases para o desenvolvimento } \\
\text { publicações atualizadas. }\end{array}$ \\
\hline Critérios para qualificação & $\begin{array}{c}\text { Os artigos escolhidos devem ser de fontes } \\
\text { confiáveis como revistas e congressos } \\
\text { reconhecidos. }\end{array}$ \\
\hline Artigos Selecionados & Artigos relacionados diretamente com o tema e \\
propósito do artigo.
\end{tabular}

Fonte: Elaboração própria (2019)

Gráfico 1 - Gráfico com critérios e informações para seleção de artigos

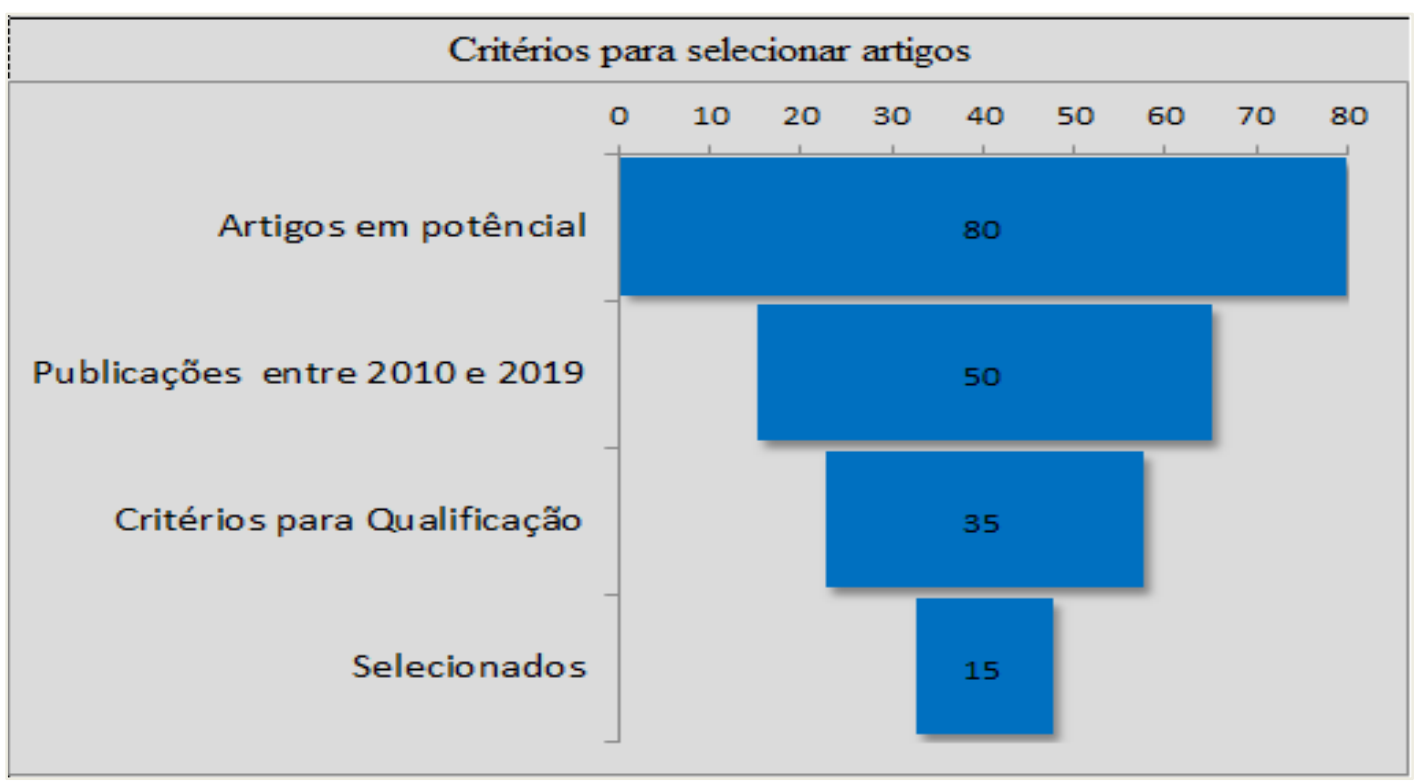

Fonte: Elaboração própria (2019) 


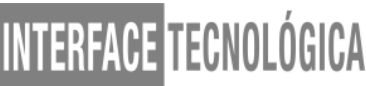

\section{RESULTADOS E DISCUSSÃO}

Conforme aferido na literatura apresentada, a proposta do estudo deste artigo foi demonstrar um parecer técnico sobre a importância da gestão por processos com a intenção fornecer conhecimento técnico científico dando-lhes conhecimento real efetivo de controle total e auxiliando na atenção em gerir seus processos de modo eficaz, em consequência disso podendo manifestar meios de concatenação a modo de conquistar uma melhor gestão e como consequência, notabilidade em meio aos concorrentes diretos no mercado, tendo em vista que com a adequação e padronização dos processos tende-se a um aumento exponencial variável no índice de conformidades nos produtos e processos, contribuindo para aceitação e credibilidade no mercado.

Apesar de que os custos diretos e indiretos para a implantação sejam muitas vezes elevados, podendo variar de organização para organização levando em consideração as necessidades da empresa e dimensão estrutural envolvendo investimentos em treinamento, consultorias, alteração de layout e da cultura organizacional por parte da resistência humana, uma força endógena predominante a mudanças envolvendo diretamente o desempenho empresarial.

Contudo ao analisar de modo holístico os benefícios adquiridos de forma natural em decorrência à decisão do nível estratégico de adotar uma gestão por processos como beneficiação, proporcionando ganhos na qualidade do produto, manuseio, armazenamento, eficácia e produtividade nas empresas.

Uma boa gestão de processos pode aferir a segurança estrutural de modo a minimizar a imprecisão do processo evitando e prevenindo gastos pela manipulação incorreta dos insumos e produtos, proporcionando um emolumento à organização podendo maximizar a produtividade e aumento do lucro.

\section{CONCLUSÃO}

Partindo dos conceitos fundamentados pela literatura estudada o presente artigo pode contribuir de modo direto a afins técnicos podendo auxiliar de maneira técnica a gestão por processos dentro de indústrias de tal modo essa gestão deve ser aplicada de forma racional e eficaz por parte do nível estratégico, agregando melhores resultados que diferencie a empresa dos demais concorrentes. 


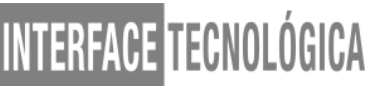

Levando em consideração o atual cenário em que as empresas se encontram e necessitam de melhorias e adequações dos processos para se destacar em meio ao mercado competitivo.

Apesar da necessidade de aplicabilidade de investimentos em alguns casos significativos por parte da empresa o modelo de gestão de processos pode ser uma ferramenta adotada como medidas que garantem possam garantir um coeficiente de segurança indispensável para que a empresa se destaque em meio ao mercado competitivo, e se caso tais medidas forem ignoradas pode ocorrer que se conduza a comprometer a estabilidade da empresa no mercado, gerando riscos com praticável possibilidade de perda de capital.

\section{REFERÊNCIAS}

ARMISTEAD, C., PRITCHARD, P. "Gestão estratégica de processos de negócios para eficácia organizacional”, Planejamento em Longo prazo, v.32, p.105-106, 1999.

BIDER, I; BELLINGER, G.; PERJONS, E. Modelando uma empresa ágil: reconciliando sistemas e pensamento de processo. Conferência de trabalho sobre a prática da modelagem de empresas v 92 p. 238-252, 2011.

DREYFUSS, Cassio. As redes e a gestão das organizações. Rio de Janeiro: Guide, 1996.

DOEBELI, G., FISHER, R., GAPP, R.; SANZOGNI, L. Usando a governança do BPM para alinhar sistemas e práticas. Jornal de gerenciamento de processos de negócios. V.17, p.184-202, 2011.

FREITAS; PRODANOV. Metodologia do Trabalho Científico: Métodos e Técnicas da Pesquisa e do Trabalho Acadêmico. 2. Ed. Novo Hamburgo: Universidade Feevale, 2013.

PAIM, Rafael; CARDOSO, Vinicius; CAULLIRAUX, Heitor. Gestão de Processos: Pensar, Agir E Aprender. São Paulo: Artmed Editora S.A., 2009.

GONÇALVES, José Ernesto Lima. Os novos desafios da empresa do futuro. RAE Revista de Administração de Empresas. V. 37, n. 3, p. 10-19, jul./set. 1997.

KANTER, R. M. Thriving Locally in the Global Economy. Harvard Business Review. p. 151158, set/oct, 1995.

HAMMER, M.; STANTON, S. How process enterprises really work. Harvard Business Review, v. 77, n. 6, p. 108-118, 1999.

HAMMER, Michael; CHAMPY, James. Reengenharia da corporação. New York: HarperBusiness, 1994. 


\section{WWTEREFEET TEENOLÓGICA}

HARMON, P., WOLF, C. (2012), O estado do gerenciamento de processos de negócios, BPTrends.

JACKSON, M. Abordagens de Sistemas à Administração. Kluwer Academic Plenum Publishers, Nova York,2000.

KERZNER, HAROLD, Gerenciamento de Projetos - Uma Abordagem de Sistemas ao planejamento, agendamento e controle. Nova York NY, John Willey \& Sons, 2001.

LAKATOS, E. M.; MARCONI, M. A. Metodologia do trabalho científico: procedimentos básicos, pesquisa bibliográfica, projeto e relatório, publicações e trabalhos científicos. 5 . ed. São Paulo: Atlas, 2001.

PRADO, DARCY. Gerenciando Projetos nas Organizações. Belo Horizonte MG, EDG, 2000.

PIERCY, N., CALDWELL, N. e RICH, N. Considerando a conectividade em periódicos de operações. International Journal of Productivity and Performance Management. V. 58, p. 607-631, 2009.

OLIVEIRA, D. P. R. (2011), Sistemas, organizações e métodos: uma abordagem gerencial. $7^{\mathrm{a}}$ ed., Atlas, São Paulo, SP.

SLACK, N.; CHAMBERS, S.; JOHNSTON, R. Administração da produção. Tradução de Henrique Luiz Corrêa. 3. Ed. São Paulo: Atlas, 2009.

TRKMAN, P. Os Fatores Críticos de Sucesso do Gerenciamento de Processos de Negócios. Revista Internacional de Gestão de Informação, v.30, p. 125-134, 2010.

YIN, R.K., Estudo de caso: planejamento e métodos. 2 . ed. Porto Alegre: Bookman, 2001.

YIN, Robert. Pesquisa Estudo de Caso: - Desenho e Métodos. 2. ed. Porto Alegre - Sc: Bookman, $1994 . \quad 173 \quad$ p. 1 Disponível <http://maratavarespsictics.pbworks.com/w/file/fetch/74440967/3-YIN-

desenho\%20e\%20metodo_Pesquisa\%20Estudo\%20de\%20Caso.pdf $>$. Acesso em: 21 jul. 2019. 